\title{
Application of Damage Control Neurosurgery and Corresponding Nursing Strategy in Patients with Bilateral Frontal Lobe Contusion and Laceration
}

\author{
Huixuan Chen ${ }^{1, \dagger}$, Yanhong Xie ${ }^{1, \dagger}$, Weiju Chen ${ }^{2, *}$, Cuiqing Liu ${ }^{3}$, Jiaming Wu ${ }^{1}$, Keen Chen ${ }^{1}$ \\ ${ }^{1}$ Department of Neurosurgery, The First Affiliated Hospital of Jinan University, Guangzhou, China \\ ${ }^{2}$ School of Nursing, Jinan University, Guangzhou, China \\ ${ }^{3}$ Department of Surgery, The First Affiliated Hospital of Jinan University, Guangzhou, China
}

Email address:

chenweiju@126.com (Weiju Chen)

${ }^{*}$ Corresponding author

$\dagger$ Huixuan Chen and Yanhong Xie are co-first authors.

\section{To cite this article:}

Huixuan Chen, Yanhong Xie, Weiju Chen, Cuiqing Liu, Jiaming Wu, Keen Chen. Application of Damage Control Neurosurgery and Corresponding Nursing Strategy in Patients with Bilateral Frontal Lobe Contusion and Laceration. Clinical Neurology and Neuroscience. Vol. 4, No. 2, 2020, pp. 24-28. doi: 10.11648/j.cnn.20200402.11

Received: March 2, 2020; Accepted: April 1, 2020; Published: April 23, 2020

\begin{abstract}
Objective: To investigate the key points and effect of damage control neurosurgery (DCNS) and nursing strategy of patients with neurosurgery in bilateral frontal contusion. Methods: According to the principle of damage control nursing strategy of neurosurgery, 33 cases of bilateral frontal contusion from September 2017 to January 2019 were treated by adjusting the internal environment disorder after trauma, increasing plasma osmotic pressure and maintaining blood oxygen saturation, craniotomy and decompression operations when patients grew worse. Patients were followed up for 6 months with the corresponding nursing practice given to the patients out of hospital. Results: Among the 33 cases, 25 of them received successful conservative treatment, 8 were converted to surgery. The operation rate was $24.2 \%$. Six months after treatment, there were 27 cases with GOS $\geq 4$, including 22 cases GOS $\geq 4$ in the conservative treatment group and 5 cases GOS $\geq 4$ in the surgery transfer group. Conclusion: The application of DCNS in the treatment of bilateral frontal contusion and take appropriate nursing strategy intervention can reduce the operation rate, prevent complications and promote patient's recovery.
\end{abstract}

Keywords: Damage Control Surgery, Craniocerebral Trauma, Bilateral Frontal Contusion, Conservative Treatment, Internal Environment Homeostasis, Craniotomy, Nursing Strategy

\section{Introduction}

The concept of damage control surgery (Damage Control Surgery, DCS) was put forward by Rotondo et al [1] in 1993, its purpose is to save lives, improve the disorder of the internal environment after trauma, and perform deterministic surgery on patients who need surgery at the right time, so as to reduce the secondary blow to patients in the acute stage of trauma. Damage control neurosurgery (Damage Control Neurosurgery, DCNS) is the refinement and continuation of DCS concept in neurosurgery. Bilateral frontalis contusion and laceration is common in acute severe craniocerebral trauma. The early condition of the patients is complex and the progress is rapid, which makes it difficult to choose the treatment plan. The application of damage control neurosurgery (DCNS) in bilateral frontolobe brain contusion and laceration can reduce craniotomy and decompression and its complications, which is beneficial to the rehabilitation of the injured [2]. How to properly apply the principle of damage control surgery to treat and nursing, and to improve the survival rate and rehabilitation of patients to the greatest extent, which poses a new challenge to nursing work.

From September 2017 to January 2019, our hospital applied the DCNS strategy to 33 patients with bilateral frontal lobe brain contusion and laceration. According to the principle of Damage Control Neurosurgery, the corresponding nursing strategy intervention was formulated and achieved good 
results. The results are as follows.

\section{Clinical Data}

Cases included criteria: head CT showed bilateral francophone brain contusion with or without hematomas, and no cerebral hernia occurred. Exclusion criteria: unilateral or bilateral pupil dilatation occurred on admission or within 6 hours after admission, or cerebral hernia caused by hematomas in other parts of the brain required emergency operation.

General information showed that there were 33 cases (M 24, F 9; age 21-67 years, mean (46.3 \pm 5.7$)$ years). The causes of injury were 12 cases of falling injury, 19 cases of car accident injury and 2 cases of others. The time of arrival department Neurosurgery of injury was 30 minutes to 2 days, Glasgow coma score (Glasgow Coma Scale, GCS): $13-15$ points in 5 cases, 9-12 points in 18 cases, 6-8 points in 10 cases. After admission, CT examination showed that there were some bilateral frontolobe lesions, flake high density or mixed density lesions, with or without peripheral low-density shadow. In some cases, the forehead angle of the lateral ventricle and suprasellar cistern compression became smaller and deformed.

\section{Nursing Strategy and Method}

\subsection{Emergency Care and Preoperative Nursing Strategy}

Nursing assessment: nurses in our department immediately according to the early warning MEWS score scale to identify the best critical point of severity of the disease, when the patient MEWS $>5$ points, the possibility of deterioration of the disease, when the patient MEWS $>9$ points, the risk of death increased significantly, prepare first aid. There were 28 patients in this group, GCS $<12$ points, all of them were ready for blood distribution and skin preparation. During observation, MEWS $>5$ points and GCS scores were decreased compared with the previous ones, and 5 cases with dilatation of pupil and 3 cases were found. Hematoma increased after CT examination was found.

Establishment of ECG monitoring, continuous monitoring of respiration, changes of blood oxygen saturation, keeping respiratory tract unobstructed, timely removal of blood sputum and vomit in mouth and nasal cavity, adequate oxygen supply, regular blood gas analysis, continuous monitoring of $\mathrm{SO} 2(\mathrm{SO} 2,>94 \%)$. If the partial pressure of oxygen in arterial blood is still low under the condition of maximum oxygen supply, mechanical assisted respiration should be given as soon as possible. The concentration of oxygen increased or decreased depending on the results of $\mathrm{SO} 2$ and arterial blood gas analysis. Mechanically assisted breathing patients strengthened airway management and timely sputum aspiration. The arterial blood was collected twice a day for blood gas analysis. The oxygen content and acid-base balance of arterial blood in the patient were examined. According to the doctor's orders, the arterial oxygen partial pressure of blood lactic acid (LAC) $2 \mathrm{mmol} / \mathrm{L}, \mathrm{pH}$ 7.35 7.45, was adjusted to above $60 \mathrm{mmgh}$. For patients with dyspnea with GCS $<8$ points and skull base fracture at admission or during treatment, doctors should be assisted in establishing artificial airway, using synchronous ventilator to assist breathing, doing good airway nursing, sucking sputum in time, fixing tracheal intubation to prevent unplanned exudation. In this group, 12 patients had indwelt tracheal intubation for 3-10 days, one patient needed ventilator-assisted breathing, no ventilator-associated pneumonia and unplanned exudation.

To ensure effective circulating blood volume, to establish more than 2 venous channels, 1 to correct acid-base balance and electrolyte disorders and effective antibiotics, to create conditions for surgery. Routine central vein catheterization. According to the central venous pressure, the volume and speed of rehydration were adjusted to monitor the range of CVP $8 \sim 12 \mathrm{~cm} \mathrm{H}_{2} \mathrm{O}$, continuous invasive arterial pressure monitoring to maintain the average arterial pressure at about $80 \mathrm{mmhg}$ to ensure intracranial perfusion, accurate recording of input and exit, observation and recording of urine volume per hour, it was found that the urine volume per hour exceeded $250 \mathrm{ml} / \mathrm{h}$ without dehydrating agent, we should be alert to the occurrence of diabetes insipidly. It was noted that urine volume was less than $20 \mathrm{ml} / \mathrm{h}$. If blood pressure increases and respiratory rhythm changes, we should beware of intracranial hypertension sign, check pupils every half hour and find the change of condition in time. Prepare breathing airbags and rescue drugs by the bed so that items can be readily picked up. In this group, 5 cases of hypovolemic shock occurred in the new admission hospital. Patient's CVP4-6 $\mathrm{cm} \mathrm{H}_{2} \mathrm{O}$ had stable blood pressure after timely infusion expansion and continued conservative treatment.

Select broad-spectrum antibiotics combined with drugs to effectively control infection. Patients with high fever were treated with mild hypothermia every 2 hours to check their skin compression and whether they had frostbite or not, and those with low temperature were treated with covered quilts to keep warm. There is no infection, no frostbite in this group.

Prevention of seizures: sodium valproate was continuously pumped into the vein to assist doctors in drawing blood and monitoring blood concentration: 50-100 $\mu \mathrm{g} / \mathrm{ml}$. Keep the ward and quiet, the light is soft, and reduce unnecessary stimulation.

In irritable patients, sedation was used as directed by the doctor, sedation score was performed on each shift, and the sedation level was controlled at Rassay score 3-4.

Keep safety sending out to CT examination, use the transport safety verification form, evaluate the patient's situation, prepare the oxygen bag, respiratory airbag, rescue items according to the patients' condition, go out for CT examination, and prepare the hospital elevator In time to reduce the waiting time for the patient. All 33 patients were subjected to CT examination 1-3 times without any adverse events.

\subsection{Post-operation Nursing}

\subsubsection{Psychological Nursing}

In this group, 8 patients were transferred to surgical treatment during DCNS treatment, and the patients were faced 
with operation, which could lead to different degrees of tension, anxiety, fear and so on. Nurses should actively communicate with patients and their families, explain the necessity of nursing operation, treatment and operation, introduce successful cases, establish patients' confidence, stabilize their mood, and better cooperate with treatment and nursing.

\subsubsection{Prevent Complications}

Continue to use antiepileptic drugs according to preoperative, administered sodium valproate intravenous continuous pumping, the first dose of $800 \mathrm{mg} 0.9 \%$ saline 20 $\mathrm{ml}$ intravenous injection, continue to use sodium valproate $12000.9 \%$ saline $50 \mathrm{ml}$, at a speed of $2 \mathrm{ml} / \mathrm{h}$ slow intravenous pumping. No seizures occurred in this group. There were 6 cases of postoperative irritability, $0.4 \mathrm{mg}$ saline $50 \mathrm{ml}$ of dexmedetomidine injection was used as prescribed by the doctor, and the patients were given acid-producing drugs to prevent stress ulcer. Three patients with multiple chronic diseases, so strengthen monitoring, improve heart, lung, liver, kidney function, prevent heart failure, kidney failure and other complications, maintain electrolyte and acid-base balance. Closely observe the changes of consciousness, pupil, vital signs, and early detection of brain swelling or intracranial re-bleeding symptoms. There was no intracranial hemorrhage after operation.

\subsubsection{The Nursing Care of the Drainage Tube}

Properly fixed and marked, the drainage is kept unobstructed, the color, quality and quantity of drainage fluid are accurately recorded, the sterile operation is strictly observed, and the drainage bag is replaced regularly. Observe the indication of pipe intubation and excitation in time.

In 8 patients after operation, the epidural drainage tube was removed 24 hours after operation, and the drainage fluid was about $60-240 \mathrm{ml}$ blood fluid.

\subsubsection{Continuous Monitoring of Intracranial Pressure}

Records the intracranial pressure of patients every hour, properly fix the wire of the induction probe of intracranial pressure monitoring to prevent accidental prolapse. If it is found that the monitoring data of intracranial pressure has been unchanged, we should check whether the intracranial pressure probe has emerged, and all cases of intracranial pressure monitoring in this group have no accidental prolapse.

\subsubsection{Nutritional Support and Nursing Patients}

Because of surgical trauma, long time cannot eat through the mouth, consumption is very large, there are different degrees of negative nitrogen balance. Individualized enteral and parenteral nutrition programs should be formulated depending on the patient's condition. Abdominal symptoms were closely observed during parenteral nutrition and changes in electrolyte. Blood ammonia and blood sugar were monitored. All 33 patients were admitted with NRS-2002 nutrition score 3-9, all of them were nutritionally risky, according to the doctor's instructions, using $10 \%$ fat milk, amino acid and other venous nutrition support, 28 patients who could not eat through the mouth after injury, after 24 hours of admission, were indwelling gastric tube through the nose to pump in $500 \mathrm{ml}$ QD or bid with $30 \mathrm{ml} / \mathrm{h}$ uniform nasal feeding, gradually increasing the amount and speed of nasal feeding, and slowly transitionally to $50-80 \mathrm{ml} / \mathrm{h}$ for full nasal feeding. A patient with ventilator-assisted breathing was given indwelling nasal and intestinal tube nasal feeding to prevent regurgitation and ventilator-associated pneumonia.

\section{Results}
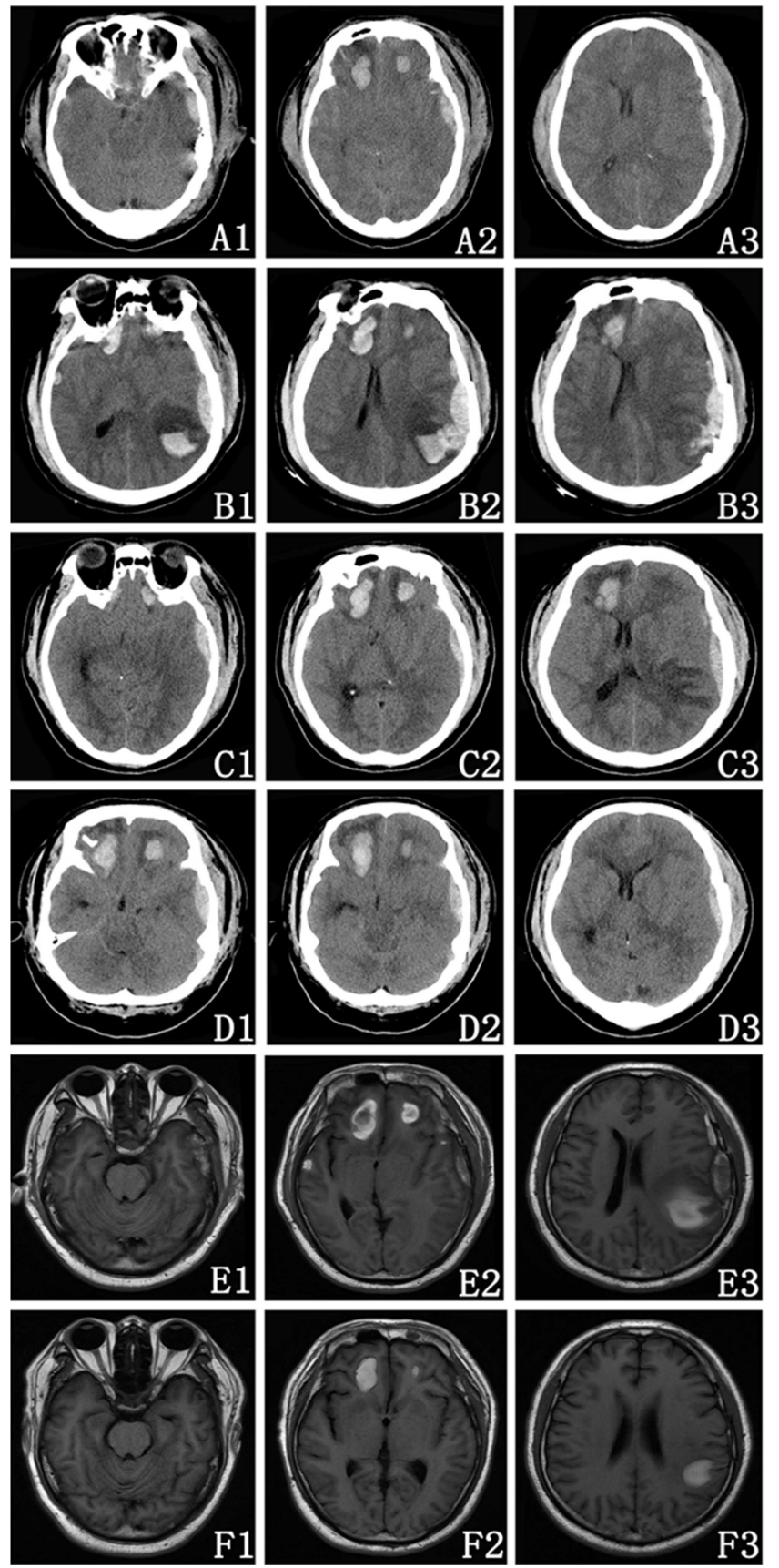

Figure 1. CT and MRI images of typical cases treated conservatively.

A-C: CT at day 1 to 3 after admission, D: CT at day 5; E, F: MRI at day 10 and 411. Skull base ring cistern level; 2. Contusion and bleeding focus level; 3 . Lateral ventricle frontal angle level. 


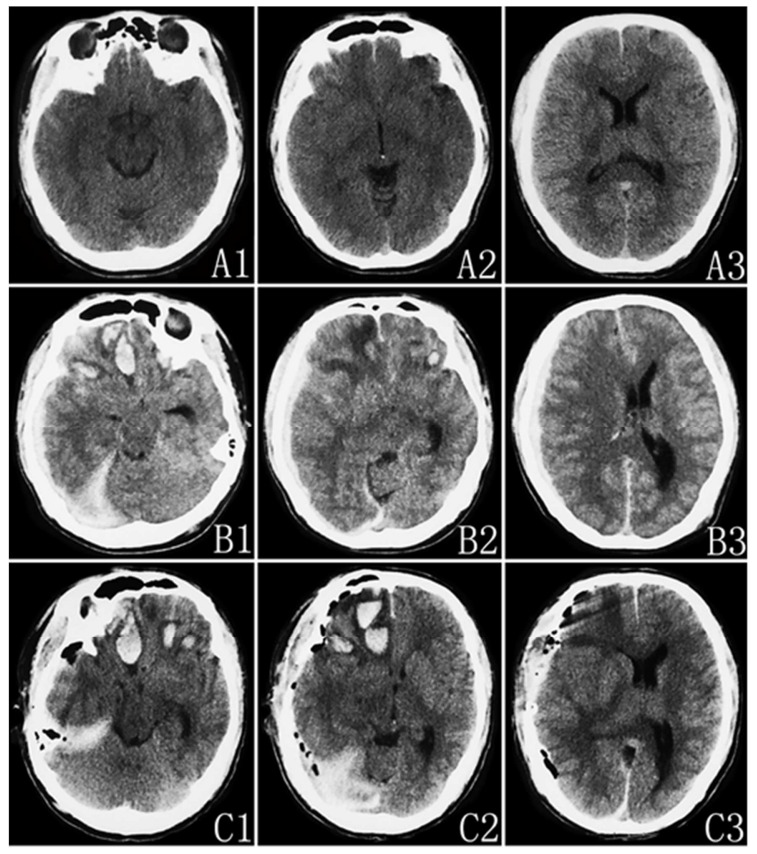

Figure 2. CT images of converted to unilateral decompression surgery group.

A-C: CT on the first day after admission, before surgery, and after unilateral decompression1. Skull base ring cistern level; 2. Contusion and bleeding focus level; 3. Lateral ventricle frontal angle level.
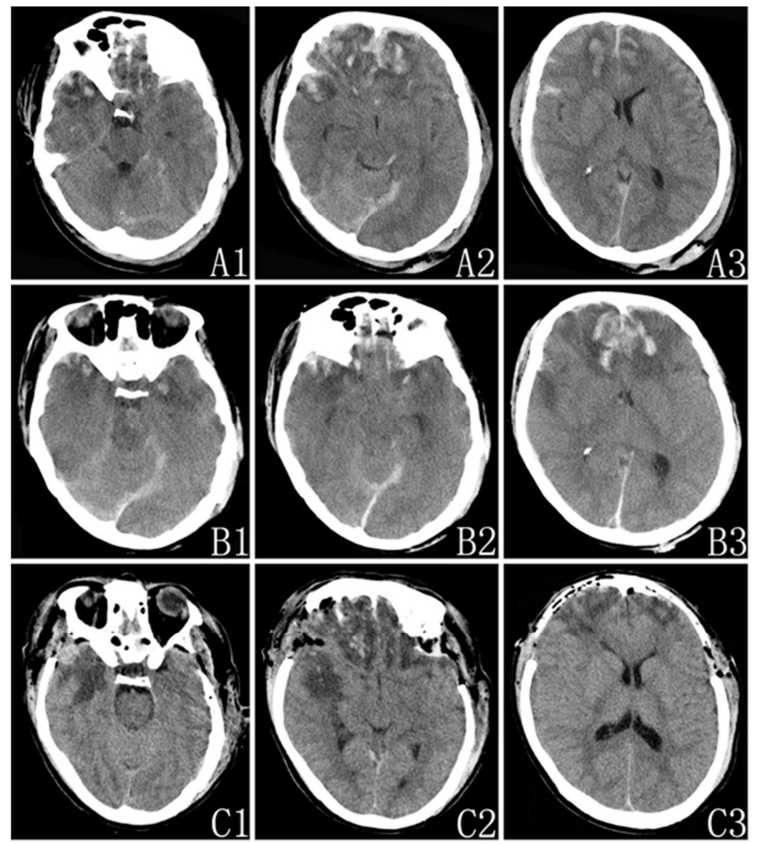

Figure 3. CT images of converted to bilateral decompression surgery group.

A-C: CT on the first day after admission, before surgery, and after bilateral decompression1. Skull base ring cistern level; 2. Contusion and bleeding focus level; 3. Lateral ventricle frontal angle level.

Among 33 cases, 25 cases (76\%) were treated conservatively (Figure 1), 8 cases $(24 \%)$ were transferred to operation, including 5 cases of unilateral operation (Figure 2) and 3 cases of bilateral operation (Figure 3 ). Six months after follow-up, Glasgow prognosis score (Glasgow Outcome Scale, GOS): 11 cases recovered well, 16 cases had mild disability, 4 cases had severe disability, 2 cases survived, 0 cases died, the total mild disability and good rate were $81.8 \%$. In the conservative treatment group, there were 9 cases of good recovery, 13 cases of mild disability, 2 cases of severe disability, 1 case of plant survival, 8 cases of mild disability and good rate of good, 2 cases of mild disability, 3 cases of mild disability, 2 cases of severe disability, 1 case of plant survival, and the rate of mild disability and good improvement was $62.5 \%$.

\section{Discussion}

\subsection{Formation and Purpose of DCS Concept}

Damage control surgery (DCS) was initially a simplified treatment strategy for patients with severe trauma, especially complex injuries, aimed at rapidly stabilizing the body's environment, saving life and improving survival. Compared with the complex operation of complete repair, the treatment strategy of early simple operation or treatment has a better therapeutic effect. After a large sample of clinical studies proved that: blood loss, hypothermia, coagulation dysfunction, metabolic acidosis is the main cause of postoperative death in some patients in the early stage, if this is not corrected, the postoperative mortality rate is up to $90 \%$ [3], so emphasis is placed on further surgical treatment after this correction. The principle of DCS is to try not to aggravate the secondary damage of the patient, to give the injured person appropriate treatment at the appropriate time to improve their survival rate and to maximize their physiological, pathological and social functions [4].

\subsection{Application of DCS in Bilateral Frontal Lobe Contusion and Laceration}

In recent years, DCS has gradually expanded from early concentration of abdominal trauma to the clinical rescue of orthopedics, traumatic brain injury, especially multiple injuries, and has made some progress [5-8]. Damage Control Neurosurgery (DCNS) is the extension and centralization of DCS in neurosurgery. In the past, DCNS focused on improving surgical methods, reducing surgical trauma and not paying enough attention to the change of the systemic environment. With the gradual improvement of DCS concept, DCNS should not only pay attention to the operation, but also pay attention to the comprehensive treatment strategies such as evaluation technology, monitoring method, operation time, operation mode, postoperative treatment and so on. Some studies have shown that [9], the improvement of the level of treatment of severe traumatic brain trauma depends on the progress of monitoring means, including intracranial pressure monitoring, hemodynamic monitoring, brain oxygen monitoring, brain temperature monitoring, brain imaging monitoring and so on. In the use of these monitoring means of nursing monitoring plays an important role, accurate assessment of the disease, timely detection of patient's disease changes. This period should provide the necessary preparation for the deterministic operation, for the patients to buy the operation time, in this 
group of cases we implement the corresponding nursing measures, closely cooperate with the doctor, timely found 5 patients due to re-bleeding after trauma caused by one side of the pupil dilatation, timely operation, turn to safety. Three patients re-examined $\mathrm{CT}$, found hematoma enlargement to surgery. How to choose more valuable monitoring methods in different treatment environments is the key to the development of a treatment plan.

A multicolor study from abroad showed that craniocerebral trauma accounted for $34 \%$ of the total number of injuries, of which nearly half (49.7\%) were caused by car accidents [10]. Another multicenter study showed that the mortality rate of patients with craniocerebral trauma was as high as $60 \%$. The total mortality rate of patients with craniocerebral trauma $(18.2 \%)$ was three times higher than that of patients without craniocerebral injury (6.1\%) [11]. Reasonable treatment should be given to patients with bilateral frontalis contusion and laceration at the right time. Excessive emphasis on the operation does not necessarily bring benefits to the injured. Craniotomy and decompression can be carried out after closely monitoring the changes of the condition, fully preparing, adjusting the internal environment of the whole body and so on. One day after injury, the patients were transferred to our hospital (Figure 1), GCS 6). CT showed double forehead and intracranial multiple cerebral contusion and hemorrhage, the basal cistern disappeared, the ventricle became smaller, but there was no pupil cerebral hernia, so it was difficult to choose whether to operate or not. We used DCNS principle to nurse, closely monitor vital signs, maintain the balance of the internal environment, establish an artificial airway, use synchronous ventilator and conservative treatment, the condition was gradually stable. On the 10th day after injury, the basal cistern could be seen on the 10th day after injury, the bilateral fissure pool was good, the brain structure was basically normal on the 41st day after injury, and the patient recovered well.

According to the principle of injury control surgery, the treatment strategy of gradual transition from simple operation to complex operation was used to treat patients with bilateral frontalis contusion and laceration, which could significantly reduce the rate of craniotomy and its secondary injury, which was similar to that of our study (24\%). On the third day after injury, the cerebral hernia was treated with left craniotomy and decompression due to delay left frontotemporal subdural hematomas (Figure 2). GOS 5 points 6 months later, bilateral craniotomy decompression was performed on the 5 th day after injury (Figure 3) and GOS 3 points 6 months later. Surgical decompression within half an hour of cerebral hernia can effectively save life and avoid further damage of nerve function. The prognosis of the injured may be more closely related to primary brain injury such as brainstem injury and extensive brain contusion and laceration [12]. From the process of this study, the number of cases in this study is relatively small, and the results need to be further improved.

In a word, the application of DCNS concept to the treatment of patients with bilateral frontolobe brain contusion and laceration, closely observing the changes of the disease, actively adjusting the balance of the internal environment, and giving reasonable treatment at the right time can reduce the operation rate and reduce its complications, so as to benefit the patients and have important application value. The implementation of DCS, nursing observation throughout the whole, also has great challenges to the corresponding nursing work, nurses should understand the concept and connotation of DCNS. The nursing intervention strategy was worked out to deal with it positively.

\section{References}

[1] Rotondo M F, Schwab C W, Mcgonigal M D, et al. 'Damage control': an approach for improved survival in exsanguinating penetrating abdominal injury [J]. J Trauma, 1993, 35 (3): 375-382, 382-383.

[2] Xu Qinyi, Dong Jirong, Wang Yuhai, et al. Damage control surgery for bilateral frontal cerebral contusion and intracerebral hematoma [J]. Chin J Minim Invasive Neurosurg, 2015, 20 (9): 402-404.

[3] CHANG Tao, LI Li-hong. Surgical Concept of Damage Control and Its Application in Treatment of Traumatic Brain Injury [J]. Medical Recapitulate, 2015, 21 (15): 2758-2760.

[4] Clinical misdiagnosis \& Mistherapy 2014, 27 (07): 35-37.

[5] Zhou Fang, Yang Xiaoning. Observation on the effect of injury control and nursing cooperation in severe head injury [J]. Journal of Nurses Training, 2015, 30 (04): 334-335.

[6] Xiao-lan, HUANG Chun-yu, et al. Application of damage controlled snrgery and nursing in abdominal serious [J]. Chin J Mod Nurs. 2012, 18 (3): 293-295.

[7] Li Chengxiang, Xie Kegong. Rescue and Nursing of Severe Multiple Fractures with Injury Control Technology [J]. Youjiang Medical Science, 2009, 37 (05): 623-624.

[8] Liu Jianshuang, Zhu Xiaoyu, Zheng Yufang. Application of injury control in nursing of critically ill patients in orthopedics [J]. Journal of Traditional Chinese Medicine Management, 2015, 23 (06): 91-92.

[9] Feng Hua, Li Fei, Zhu Gang, et al. Application of injury control theory in the treatment of patients with severe head injury [J]. Chinese Journal of Traumatology, 2010, 26 (10): 865-867.

[10] Gennarelli T A, Champion H R, Copes W S, et al. Comparison of mortality, morbidity, and severity of 59,713 head injured patients with 114,447 patients with extracranial injuries [J]. J Trauma, 1994, 37 (6): 962-968.

[11] Gennarelli T A, Champion H R, Sacco W J, et al. Mortality of patients with head injury and extracranial injury treated in trauma centers [J]. J Trauma, 1989, 29 (9): 1193-1201, 1201-1202.

[12] Yuan Jie, Huo Chunfen. Study on prognosis status and the influential factors of severe craniocerebral trauma [J] Chinese Journal of practical Nervous Diseases, 2014, 17 (01): 3-6. 